\title{
Transmit power control and data rate enhancement in cognitive radio network using computational intelligence
}

\author{
Paurav Goel $^{1}$, Avtar Singh ${ }^{1}$, Ashok Goel ${ }^{2}$ \\ ${ }^{1}$ Department of Electronics and Communication Engineering, IK Gujral Punjab Technical University, Kapurthala, India \\ ${ }^{2}$ Department of Computer Science Engineering, MRS Punjab Technical University, Bhatinda, India
}

\begin{tabular}{l} 
Article Info \\
\hline Article history: \\
Received Mar 21, 2021 \\
Revised Sep 9, 2021 \\
Accepted Oct 4, 2021 \\
\hline
\end{tabular}

Keywords:

Bandwidth

Interference temperature

Primary user

Secondary user receiver

Secondary user transmitter

\begin{abstract}
Underutilized radio frequencies are the chief apprehension in advance radio communication. The radio recourses are sparse and costly and their efficient allocation has become a challenge. Cognitive radio networks are the ray of hope. Cognitive radio networks use dynamic spectrum access technique to opportunistically retrieve and share the licensed spectrum. The licensed users are called primary users and the users that opportunistically access the licensed spectrum all called secondary users. The proposed system is a feedback system that work on demand and supply concept, in which secondary receivers senses the vacant spectrum and shares the information with the secondary transmitters. The secondary transmitters adjust their transmission parameters of transmit power and data rate in such a way that date rate is maximized. Two methods of spectrum access using frequency division multiple access (FDMA) and Time division multiple access (TDMA) are discussed. Interference temperature limit and maximum achievable capacity are the constraints that regulate the entire technique. The aim of the technique is to control the transmitter power according to the data requirements of each secondary user and optimizing the resources like bandwidth, transmit power using machine learning and feed forward back propagation deep neural networks making full use of the network capacity without hampering the operation of primary network.
\end{abstract}

This is an open access article under the $\underline{C C B Y-S A}$ license.

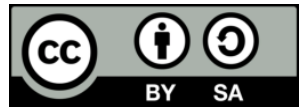

\section{Corresponding Author:}

Paurav Goel

Department of Electronics and Communication Engineering, IK Gujral Punjab Technical University Jalandhar-Kapurthala, Highway, Vpo, Ibban, Punjab 144603, India

Email: pauravg5986@gmail.com

\section{INTRODUCTION}

Currently Federal Communication Commission (FCC) in United State assigns spectrum frequency blocks [1] for a particular requirement and gives licenses to these blocks. It has been well proved by several reports that spectrum is not exploited properly or it is below utilization level. A statistic of the report presented in 2004 , revealed that utilization of spectrum is only $13 \%$. Cognitive radio technology is proving to be a promising technology in solving the problem of underutilization of valuable spectral resources. Cognitive radio (CR) is a highly flexible and low-cost radio firstly introduced by Mitola [2]. According to them CR [2] is an intelligent system that has the knowledge of environment in which it is functioning. It learns from the environment and then adapts its internal states according to the incoming stimuli and then reconfigures its transmission parameters to operate in real time. The two main objectives of CR are efficient usage of the radio spectrum and reliable communication. Cognitive radio system there are no. of secondary user (SU's) and primary user (PU's), and each SU selfishly tries to raise its transmission power to increase its own utility due to which an inevitable increase in the interference at other SU's takes place, therefore resulting in mutual 
interference between SU's and the interference of SU's with PU's. To control the problem interference temperature is the suitable metric. Here generalised method [3] of interference temperature calculation is considered. In generalized method the interference temperature model is applied to entire frequency range and not just where primary users are detected. It includes co-channel interference that is due emissions from other transmitting devices functioning on the identical frequency as the chosen transmitter and adjacent channel interference due to emissions from transmitting devices working on adjacent frequencies. For efficient and reliable use of spectrum the interference temperature has to be kept below a predefined threshold also care has to be taken that maximum capacity of the spectrum band is exploited. By proficient allocation of bandwidth and transmit power in a step-by-step manner and allocating the transmit power wisely to the secondary transmitter according to the data and $\mathrm{S} / \mathrm{N}$ requirements of secondary receiver the spectral efficiency of the overall system can be improved before the interference temperature limit is achieved.

In [4] a robust method of transmit power control in multi user cognitive radio system is considered. Control theory is used to study the balance and transient behavior of network under dynamically changing conditions. In [5] game theory method is used to control the transmit power of multi SU's in order to achieve a balance point such that minimum interference is caused to primary users. In [6] a method is investigated to set maximum allowable transmit power for secondary base station. Shadowing between secondary base station and primary user receiver as well as primary user transmitter and secondary user receiver are considered. Maximum transmit power setting in cooperative scenario outperforms that of non-cooperative scenario. In [7] fixed and adaptive power control methods are reviewed and a new method is proposed in which accurate sensing information is collected by employing more no. of antennas. Better sensing information leads to better transmit power control. In [8] a genetic algorithm is used to allocate recourses to maximize overall network capacity and optimize the power. Genetic algorithm (GA) outperforms the greedy algorithm. In [9] cognitive radio using relay network is studied. The channel efficiency and quality are enhanced using diversity techniques and weight adaptation concept to mitigate the problem of fading. In [10] various evolutionary algorithms used in cognitive radio networks are reviewed like genetic algorithm, swarm intelligence, artificial bee colony, ant colony system, cuckoo search and particle swarm optimization and their comparative evaluation is performed. In [11] medium access protocol for transmission in cognitive radio networks are studied. Centralized and decentralized cognitive radio networks are studied. The rest of paper is divided into four sections. Section 2 will discuss the proposed method. In section 3 research method is discussed. In section 4 results will be analyzed followed by conclusion and future scope.

\section{PROPOSED METHOD}

\subsection{System and frequency model}

Frequency band of $1 \mathrm{GHz}-6.5 \mathrm{GHz}$ has been considered. Primary users PU Tx1 namely with center frequency $2.5 \mathrm{GHz}$ and PU-Tx2 with center frequency $5.15 \mathrm{GHz}$ are the licensed users. 'n' secondary user transmitters SU-Tx1, SU-Tx2 SU-Txt will access the spectrum recourses of primary network to transmit message signals to $\mathrm{m}$ secondary receivers SU-x1, SU-Rx2 SU-Rxm as shown in Figure 1. The message signal will be generated using orthogonal frequency-division multiplexing (OFDM) [12], [13]. Binary phase shift keying (BPSK) modulation is used over the OFDM message signals. The arrows in the Figure 1 shows the direction of transmission. AP denotes access points and MD denoted mobile devices. A multiuser secondary network having two or more secondary transmitters will access the licensed spectrum allocated to primary users in an opportunistic way and perform communication activities. The multi secondary transmitters will access the licensed spectrum through frequency division multiple access (FDMA) [14] and time division multiple access (TDMA) [15] techniques. The implementation of both the techniques will be discussed separately. The secondary receiving units will detect the presence of Primary user through energy-based spectrum sensing [16] and inform about the vacant spectra to the secondary transmitters so that they can enable, disable or reconfigure the transmission as shown in Figure 2. The transmission parameters like bandwidth and transmission power will be allocated to each SU-Tx iteratively depending upon the input received from their SU-Rx so that communication of primary network is not hampered and there is no probability of outage [17]. The Interference temperature [18] of the entire band in which primary and secondary users are operating and the maximum allowable capacity is taken as constraints to regulate the entire process as described in Figure 2. Initially the transmission bandwidth and then the transmission power will be updated in order to minimize the difference between maximum achievable capacity [19] and the obtained data rate and the difference between the maximum allowed interference temperature limit and interference temperature achieved after all SU-Tx transmissions. The final aim is proficient allocation of resources like bandwidth and power to the SU-Tx's using machine learning and neural networks thus making full use of the network capacity without hampering the operation of primary network by keeping inside the limits of interference temperature. The proposed approach is based on demand and supply. 


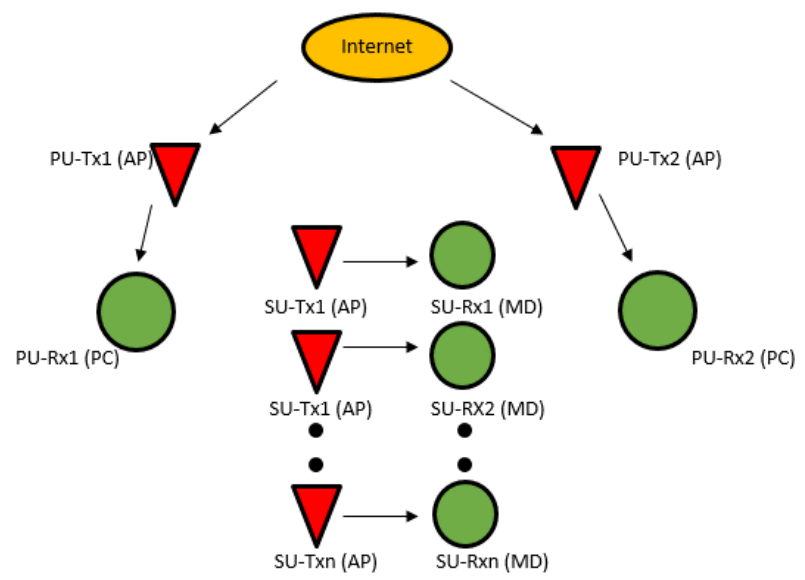

Figure 1. System model

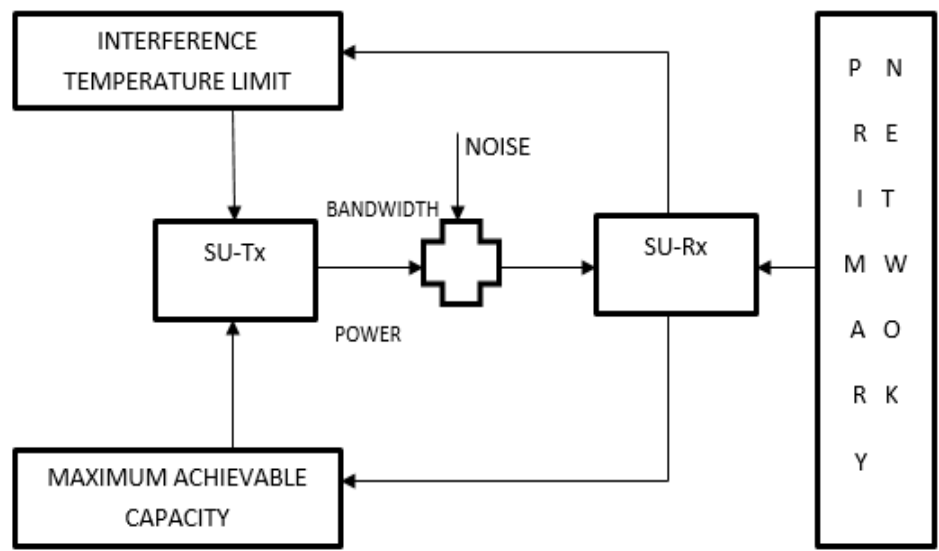

Figure 2. Feedback model

The proposed work aims to utilize machine learning (ML) in order to differentiate average and below average performance. It has been observed that, when the network is stretched to a limit when the band allocation requires high amount of computation, it fails and delivers a deliberate failure. In order to overcome the failure, the proposed work model is divided into two sections viz. the primary setup and the adjustment set. The role of the primary set up is to establish a simulation-oriented data structure which could be passed to the work mechanism acting in the second block viz. the adjustment set. The overall structure can be explained by using Figure 3. There are certain steps in the first processing block.

Step 1: The PUs prepares their data for the transmission utilizing the sweet spots of the available bandwidth. Step 2: The data is passed to the channel. To be specific in case of proposed work, three different channels viz. Additive white Gaussian noise (AWGN), Raleigh fading and Nakagami ( $M=2)$ has been used to check the robustness of the proposed algorithm

Step 3: The receiver end collects the signal passed through the channel and performs set of operations viz. Power spectral density (PSD) analysis, application of band pass filter, summing up the data with the integrator and then performing the decision threshold to analyze that the received signal is from the PU only. This step is also referred as energy detection from band spectrum.

Step 4: The energy is calculated using (1).

$$
E_{\text {con }}=\sum_{i}^{m}\left|P_{s d} m\right|^{2}
$$

where $m$ is total number of received spectrum.

Step 5: This step calculates the interference temperature (IT), data rate and maximum achievable capacity by using (2), (3) and (4). The calculation requires the carrier frequency (fc) and the channel bandwidth (B). 


$$
T_{I}\left(f_{c}, B\right)=\frac{P_{I}\left(f_{c}, B\right)}{k B}
$$

where $\mathrm{b}$ is Boltzmann Constant which is equal to $1.38 \times 10-23 \mathrm{~J} / \mathrm{K}$

$$
\mathrm{Di}=\frac{B i}{N i} \log 2\left(1+\frac{P i \mid h(i) \iota^{2}}{N o+\frac{B i}{N i}}\right) \mathrm{bits} / \mathrm{sec}
$$

$\mathrm{Ciav}=B i \log 2(1+P i / \mathrm{Ni}) \mathrm{bits} / \mathrm{s}$

$P i$ is the signal transmit power and $B i$ is the bandwidth of channel in $\mathrm{MHz}$. The process repeats itself until the maximum temperature is not attained.

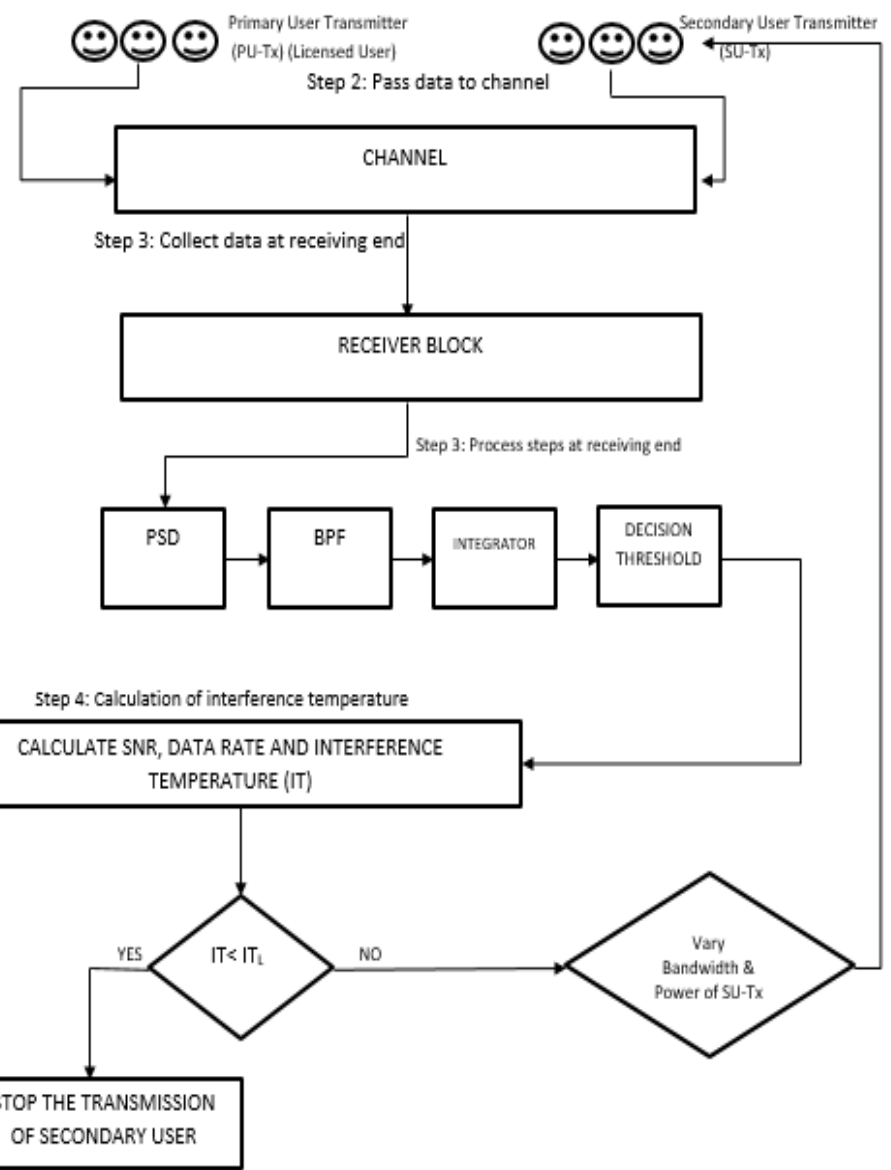

Figure 3. Block diagram of first processing block

The second block of the proposed algorithm uses a feedback mechanism over the learning architecture of machine learning. The proposed algorithm observes the performance of the time frames when the data has been transferred through observed PU data transfer and SU data transfer. A repository of 100,000 simulations analyzing the data to be on true side or on the false side is created. To avoid manual observations in the future, the simulation set was divided utilizing machine learning considering the interference temperature (IT), evaluated bit error rate (BER) and data transfer rate (Dtr) in Mbps. The simulation set was big and hence to divide the data into observed. The learning mechanism utilizes iterative k-means with an enhancement where it tends to add possibilities in the simulated dataset for the processing of division. The proposed algorithm considers channel distortion as a serious issue and hence creates a time lap of 100 units based on the current selected data. Each data unit will have a set of IR, BER, and Dtr. The proposed algorithm varies the data with ' $\mathrm{dt}$ ' amount of variation considering the average value of the current set. If one metric set is represented by Ms1, then the first variation would be Ms1+dt which would produce Ms2. Ms3 will be produced by utilizing Ms1 and Ms2 by setting dt as a random change to the neutralized attribute value of Ms1 and Ms2. This nature 
of variation is inspired by Fibonacci series. The divided part is then passed to feed forward back propagation deep neural networks [20] as shown in Figure 4. The proposed algorithm divides the data into three categories or labels as "PU++" (only PU present), "PU+SU+" (both PU and SU are present and contributes equal amount of data) and "SU++" (only SU data is present). The categories are defined in Table 1. The proposed algorithm is simulated on matrix laboratory (MATLAB) and hence using k-means was used utilizing machine learning toolbox from MATLAB. The learning toolbox divides the data into four segments with a tag of 1, 2, 3, and 4 .

Table 1. Parameters of PU's

\begin{tabular}{ccccc}
\hline User & Bandwidth (MHz) & Power & IT in dB kelvin & Data rates in Mbps \\
\hline PU1 & $40 \mathrm{MHz}$ & $60 \mathrm{~dB}$ & 440 & 72 \\
PU2 & $20 \mathrm{MHz}$ & $50 \mathrm{~dB}$ & 223 & 54 \\
\hline
\end{tabular}

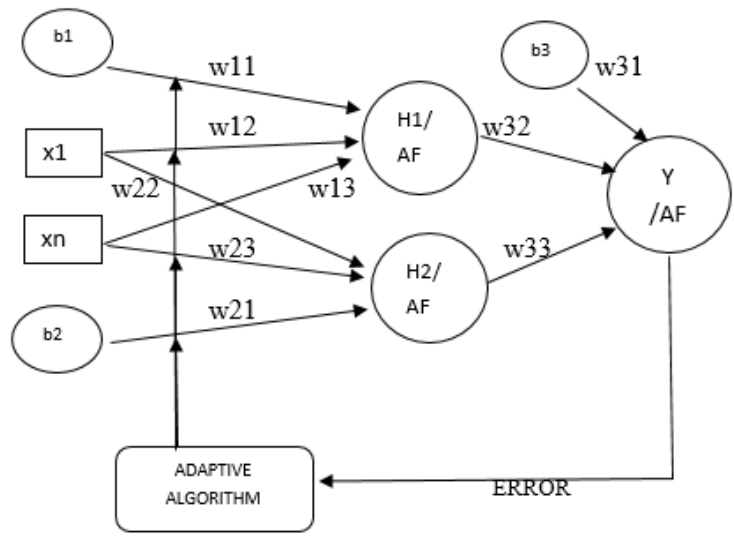

Figure 4. Block diagram of feed forward back propagation deep neural networks

To label them, it is quite necessary to find the best co-relation among the distributed members. The PU's are authenticated licensed user and hence data spectrums received by PUs will be most consistent and will come at a high data transfer rate. To evaluate the co-relation, mean absolute error (MAE) and standard error (SE) along with standard deviation (Std) has been used after "Log" based neutralization. Deep neural network (DNN) uses a forward mechanism for the weight propagation and validates through the back propagation method. The pseudo code description for the algorithm is given as.

Algorithm working

Input: Simulation-data $(\mathrm{Sd})$, labels, layer-distribution $(\mathrm{Ld})$

Labels contains the values of categories and layer distribution for the deep neural network (DNN)

Step 1. For each1 cl in Labels

Step 2. Find max-co-relation between category elements by calculating cosine similarity using (5).

Step 3.

$$
\operatorname{Cos}_{s i m}=\frac{M S_{x .} M S_{y}}{\left|M S_{x}\right|\left|M s_{y}\right|}=\frac{\sum_{i=1}^{n} M S_{x_{i}} M S_{y_{i}}}{\sqrt{\sum_{i=1}^{n} M S_{x_{i}}^{2} \sqrt{\sum_{i=1}^{n} M S_{y_{i}}^{2}}}}
$$

Step 4. If the row value having attribute set with less than even a $30 \%$ margin of the current sim value, the row is not selected as suitable row for the category and it is traded off for none. The mechanism uses natural computing oriented genetic algorithm (GA).

Step 5. End For 1

Step 6. Initiate LFB-DNN with neuron range $\{20-50\}$ with the following credentials.

Step 7. Learning mechanism: Feed forward with weight variation by polynomial trade.

Step 8. Propagation Type: Gradient Oriented

Step 9. Stop condition: Time and validation checks

Step 10. Validation ratio: $\{.15, .20, .25\}$ considering at max of $85 \%$ as training and minimum of $75 \%$ data as training data. 
Step 11. Select random sample test data

Step 12. Check true positive, false positive, true negative, false negative and precision recall along classification accuracy to ensure that best training mechanism is supplied to support first block.

Step 13. Find data validation with maximum accuracy. Store to repository and reinforce when new simulation accuracy is attained

Step 14. End Pseudo.

\section{RESEARCH METHOD}

Case 1: Multiple secondary users simultaneously accessing the licensed spectral resource (FDMA)

Two secondary users transmitting simultaneously during inactivity of one of the PU are considered.

PU-Tx 1 is transmitting at $60 \mathrm{~dB}$ and PU-Tx2 is transmitting at $50 \mathrm{~dB}$ simultaneously at center $2.5 \mathrm{GHz}$ and $5.15 \mathrm{GHz}$ respectively. The PSD plot using pwelch function in MATLAB is shown in Figure 5. Using energy based spectrum sensing the two PU signals are detected as indicated in Figure 6. The interference temperature limit that can be sustained by the channel is considered to be $3500 \mathrm{~dB}$ kelvin as shown in Table 2 . Using system temperature function in MATLAB the interference temperature of the signals received is calculated from both PU is shown in Table 2. The interference temperature (IT) caused by PU1 and PU2 in total is less than achievable limit of $3500 \mathrm{~dB}$ kelvin. As a result, new secondary users have the opportunity to transmit in underlay and overlay mode. We have considered three SU's to operate in underlay mode namely SU-Tx1, SU-Tx2 and SU-Tx3 and six SU's to operate in overlay mode namely SU-Tx4, SU-Tx5, SU-Tx6, SU-Tx7, SU-Tx8 and SU-Tx9. The parameters of SU's in underlay and overlay [21] are shown in Tables 3 and 4 respectively. The Figure 7(a) depicts the PSD of all PU's and SU's transmitting in the channel.

When one of the PU i. e. PU1 gets inactive, the interference temperature falls as shown in Figure 7(b). Two users SU-Tx1 and SU-Tx2 will transmit simultaneously in the bandwidth that was previously occupied by PU1. SU-Tx1 has higher bandwidth of $10 \mathrm{MHz}$ and SU-Tx2 has lower bandwidth occupancy of $5 \mathrm{MHz}$. The subcarrier spacing (b/ni) for SUs used in this case is $19.5 \mathrm{KHz}$. While SU-Tx3 keeps transmitting with the PU2 in underlay mode as shown in Figure 7(b).

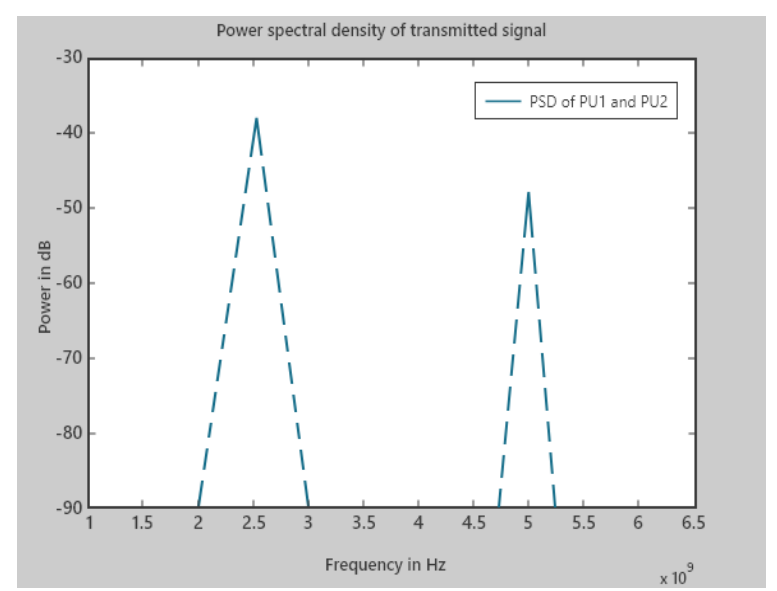

Figure 5. Power spectral density of multiple PU's

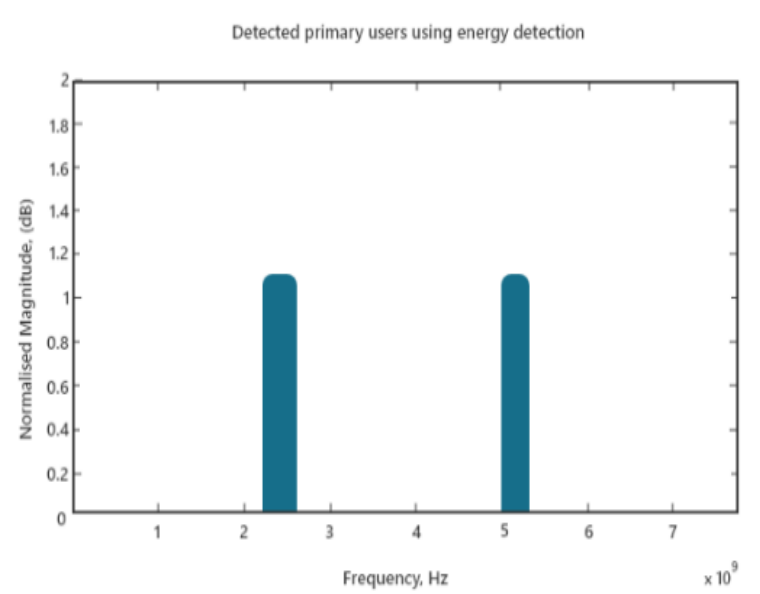

Figure 6. Output of spectrum sensing

Table 2. Interference temperature calculations of PU's

\begin{tabular}{cc}
\hline Total IT PU1 and PU2 & $663 \mathrm{~dB}$ kelvin \\
\hline Allowable IT limits & $3500 \mathrm{~dB}$ kelvin \\
IT difference & $2837 \mathrm{~dB}$ kelvin \\
Achievable capacity for PU1 & $176 \mathrm{Mbps}$ \\
\hline
\end{tabular}

Table 3. Parameters of SUs in underlay and their IT calculations

\begin{tabular}{ccccc}
\hline SU & Center frequency $(\mathrm{GHz})$ & $\mathrm{BW}(\mathrm{MHz})$ & Signal power $(\mathrm{dB})$ & IT in dB Kelvin \\
\hline SU-(Tx1) & 2.42 & 10 & 30 & 310 \\
SU-(Tx2) & 2.6 & 5 & 30 & 211 \\
SU-(Tx3) & 5.15 & 5 & 40 & 217 \\
\hline
\end{tabular}


Table 4. Parameters of SUs in overlay and their IT calculations

\begin{tabular}{ccccc}
\hline SU & CENTRE FREQ. (GHz) & BW (MHz) & POWER (dB) & IT in dB Kelvin \\
\hline SU-(Tx4) & 1.8 & 9.7 & 30 & 304 \\
SU-(Tx5) & 3.2 & 9.5 & 35 & 316 \\
SU-(Tx6) & 3.7 & 9.2 & 37 & 294 \\
SU-(Tx7) & 4.2 & 15 & 45 & 421 \\
SU-(Tx8) & 4.4 & 5 & 32 & 217 \\
SU-(Tx9) & 5.8 & 16 & 43 & 421 \\
\hline
\end{tabular}

TOTAL IT by SU-Tx1, SU-Tx2 and SU-Tx3=SU(R)=738 dB Kelvin

Total IT by SU-Tx4, SU-Tx5, SU-Tx6, SU-Tx7, SU-Tx8 and SU-Tx9=SU (B)=1973 dB Kelvin

Total IT by $\mathrm{SU}(\mathrm{R})+\mathrm{SU}(\mathrm{B})=2711 \mathrm{~dB}$ Kelvin

Total IT by PU+SU (all) $=3374 \mathrm{~dB}$ Kelvin,

IT difference $=126 \mathrm{~dB}$ Kelvin

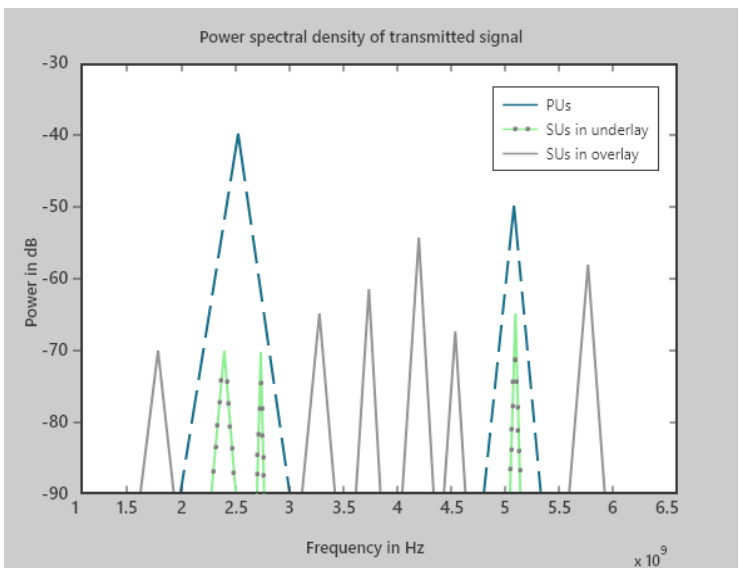

(a)

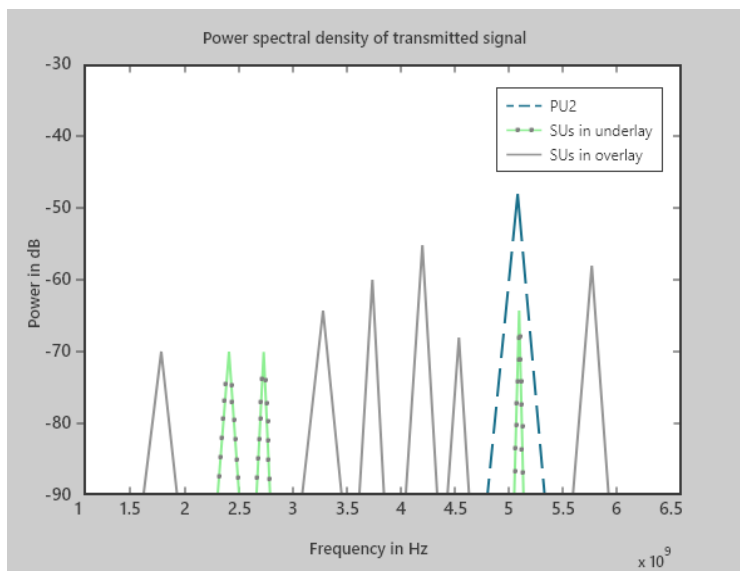

(b)

Figure 7. Power spectral density curves for all PU's and SU's in underlay and overlay accessing the spectral resources (a) PU1 is active and (b) PU1 is inactive

Firstly, the Bandwidth adaptation of SUs Tx1 and Tx2 will take place. The data rate demands of receiver of the SU-Tx1 is higher than SU-Tx2 so during inactivity of PU1, SU- R1 has faster bandwidth adaptation rate with 0.75 learning rate step, while SU-R2 has slower bandwidth (BW) adaptation rate with learning rate step of 0.5 as shown in Figure 8. Total achievable capacity of $176 \mathrm{Mbps}$ is permissible for PU1. The Table 5 shows the outcome of Bandwidth adaptation after various iterations. The Bandwidth adaptation continues till the bandwidth of both SU-Tx1 and Su-Tx2 synchronizes with original BW of PU1. It is clear that after bandwidth adaptation, the IT difference is $357.97 \mathrm{~dB}$ kelvin and data rate achieved is $122.12 \mathrm{Mbps}$. As the achievable capacity of PU1 is $176 \mathrm{Mbps}$, there is still the scope of data rate enhancement. According to Shannon theorem further enhancement in data rate can be achieved by increasing the transmit power. Further enhancement in data rate will be achieved through power reconfiguration as shown in Table 6 .

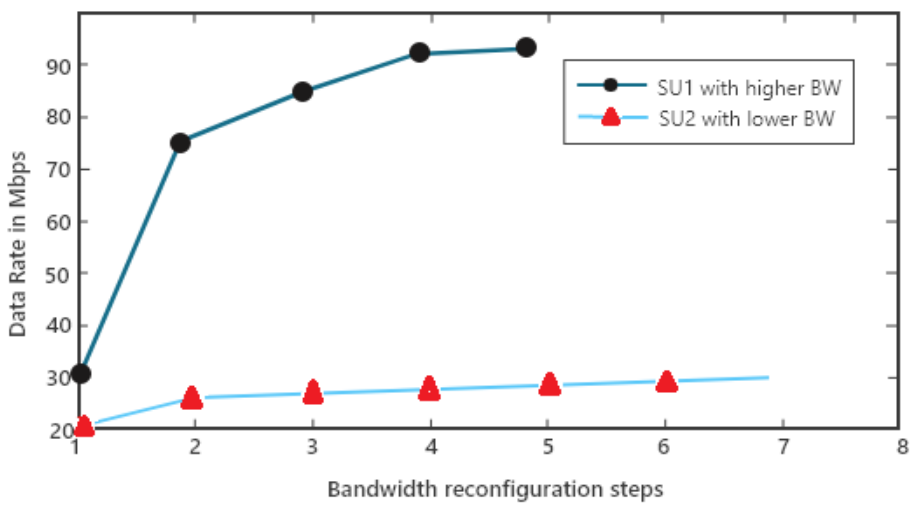

Figure 8 . Data rates achieved by SU $1^{\text {st }}$ and $2^{\text {nd }}$ after BW adaptation 
Table 5. Iterations of bandwidth adaptation of two SUs simultaneously

\begin{tabular}{|c|c|c|c|c|c|c|c|}
\hline Iteration & SU & $\begin{array}{c}\text { Reconfigured } \\
\text { BW MHz }\end{array}$ & $\begin{array}{l}\text { Power } \\
\mathrm{Db}\end{array}$ & $\begin{array}{c}\text { Data rate } \\
\text { Mbps }\end{array}$ & $\begin{array}{c}\text { IT } \\
\mathrm{dB} \mathrm{K}\end{array}$ & $\begin{array}{l}\text { Total } \\
\text { IT }\end{array}$ & $\begin{array}{l}\text { Diff. } \\
\text { IT }\end{array}$ \\
\hline \multirow[t]{2}{*}{1} & SU-Tx1 & 25 & 30 & 76.80 & 376 & 3023 & 477 \\
\hline & SU-Tx2 & 7.5 & 30 & 23.04 & 235 & & \\
\hline \multirow[t]{2}{*}{2} & SU-Tx1 & 28.75 & 30 & 88.32 & 398 & 3068 & 432 \\
\hline & SU-Tx2 & 8.75 & 30 & 26.88 & 258 & & \\
\hline \multirow[t]{2}{*}{3} & SU-Tx1 & 29.6875 & 30 & 91.20 & 418 & 3111 & 389 \\
\hline & SU-Tx2 & 9.375 & 30 & 28.80 & 281 & & \\
\hline \multirow[t]{2}{*}{4} & SU-Tx1 & 30 (SYNC) & 30 & 92.16 & 420 & 3134 & 366 \\
\hline & SU-Tx2 & 9.6875 & 30 & 29.70 & 302 & & \\
\hline \multirow[t]{2}{*}{5} & SU-Tx1 & 30 (SYNC) & 30 & 92.16 & 420 & 3138 & 362 \\
\hline & SU-Tx2 & 9.92 & 30 & 29.88 & 306 & & \\
\hline \multirow[t]{2}{*}{6} & SU-Tx1 & 30 (SYNC) & 30 & 92.16 & 421 & 3142 & 358 \\
\hline & SU-Tx2 & 10 (SYNC) & 30 & 29.96 & 310 & & \\
\hline
\end{tabular}

Total IT by SU(R) + SU (B) + PU2 = 2933 dB Kelvin, IT difference before reconfiguration= 568 dB Kelvin

Table 6. Iterations of power reconfiguration of two SUs simultaneously

\begin{tabular}{|c|c|c|c|c|c|c|c|}
\hline Iterations & $\mathrm{SU}$ & $\mathrm{BW} \mathrm{MHz}$ & Power in $\mathrm{dB}$ & Data rate mbps & IT dB K & Total IT & Diff in IT \\
\hline \multirow[t]{2}{*}{1} & SU-Tx1 & $30(\mathrm{SYNC})$ & 45 & 113.3 & 535 & 3335 & 165 \\
\hline & SU-Tx2 & 30(SYNC) & 60 & 43.1 & 389 & & \\
\hline \multirow[t]{2}{*}{2} & SU-Tx1 & 30(SYNC) & 52.5 & 121.8 & 595 & 3429 & 71 \\
\hline & SU-Tx2 & 10(SYNC) & 67.5 & 45.3 & 422 & & \\
\hline \multirow[t]{2}{*}{3} & SU-Tx1 & 30(SYNC) & 56.25 & 125.7 & 615 & 3465 & 35 \\
\hline & SU-Tx2 & 10(SYNC) & 69.380 & 45.9 & 438 & & \\
\hline \multirow[t]{2}{*}{4} & SU-Tx1 & 30(SYNC) & 58.125 & 127.5 & 624 & 3480 & 20 \\
\hline & SU-Tx2 & 10(SYNC) & 70 (sync) & 46 & 445 & & \\
\hline \multirow[t]{2}{*}{5} & SU-Tx1 & 30(SYNC) & 60 (sync) & 129.3 & 629 & 3486 & 14 \\
\hline & SU-Tx2 & 10 (SYNC) & 70 (sync) & 46 & 445 & & \\
\hline
\end{tabular}

The power reconfiguration continues until the IT difference gap is mitigated to the lowest level. The increase in transmit power will increase the range of secondary receivers and gives the flexibility to move around. The receiver of SU-Tx2 is placed at larger distance and demands of enhanced data rate, so SU-Tx2 has fast power reconfiguration. SU-Tx1 receiver requires higher data rate for longer duration at smaller range so its transmitter has low power reconfiguration. A stopping condition is attained once the IT gap is mitigated and individual data rate demands of each user is satisfied. The data rates achieved individually by two SUs are shown in Figure 9.

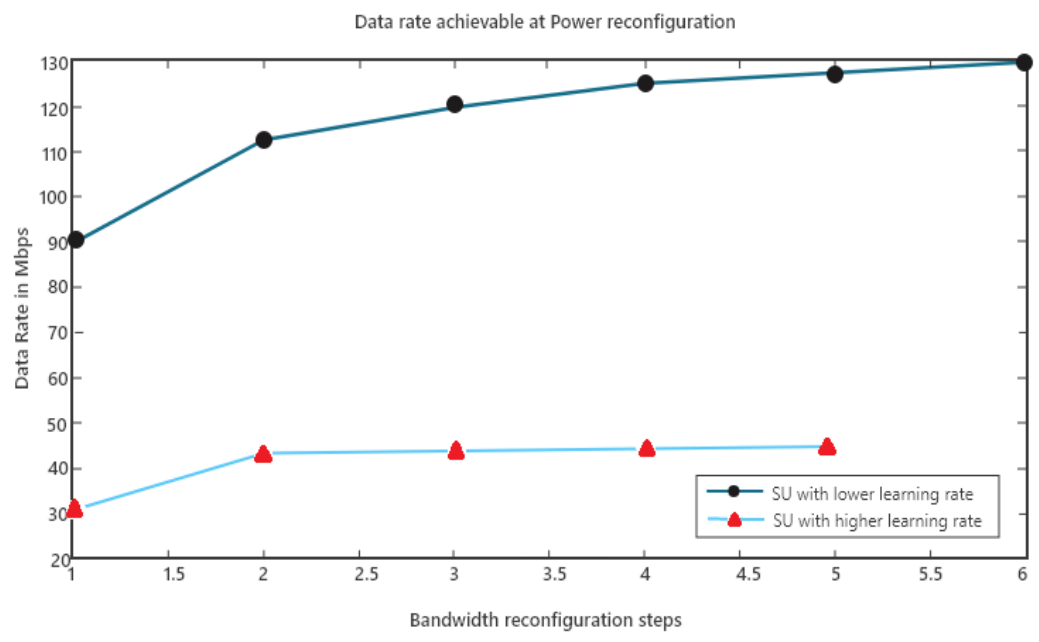

Figure 9. Data rates achieved after power reconfiguration of $1^{\text {st }}$ and $2^{\text {nd }} \mathrm{SU}$

Case 2: Multiple secondary users accessing the licensed spectrum one at a time (TDMA)

The PU1 and PU2 are operating at same parameters as discussed in case1. Due to difference in interference temperature the SU's will opportunistically access the licensed spectrum as already discussed in step 1. But now when PU1 gets inactive, the two SU's transmit at alternate time instants using the full 
bandwidth of PU1. Out of two SU's SU-Tx1 has lower data rate requirements whereas SU-Tx2 has higher data rate requirements. Both the users transmit in alternate time slot allotted to them. The simulation parameters of two SUs along with IT caused by their transmission and data rates achieved is detailed in Table 7. Firstly, the bandwidth adaptation of SU-Tx1 and SU-Tx2 takes place at their respective time slots. The individual receivers give feedback to their SU transmitters regarding IT difference and thus enables the BW adaptation of two SUs at separate time slots T1 and T2 shown by Figure 10(a) and Figure 10(b).

Table 7. Parameters of SU1 and SU2 individually and their IT calculations

\begin{tabular}{ccccccc}
\hline SU & BW MHz & Power dB & DATA RATE Mbps & Achievable capacity mbps & IT dB K & IT Diff. \\
\hline SU-Tx1 & 5 & 30 & 20.4 & 240 & 217 & 872 \\
SU-Tx2 & 10 & 40 & 95.1 & 460 & 226 & 862 \\
\hline
\end{tabular}

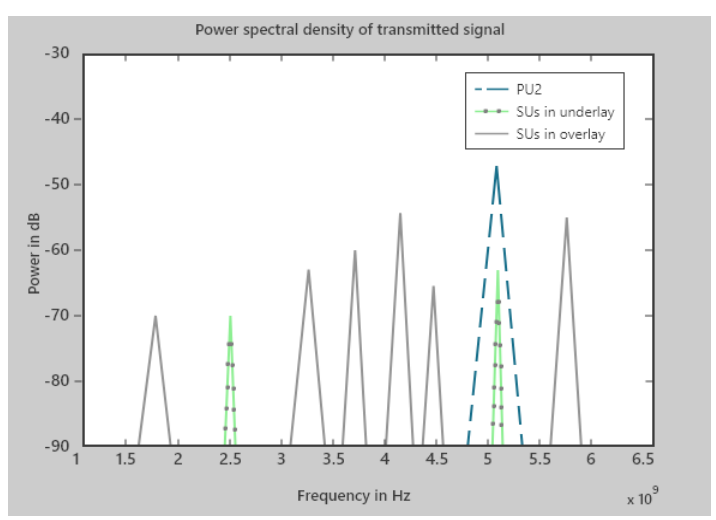

(a)

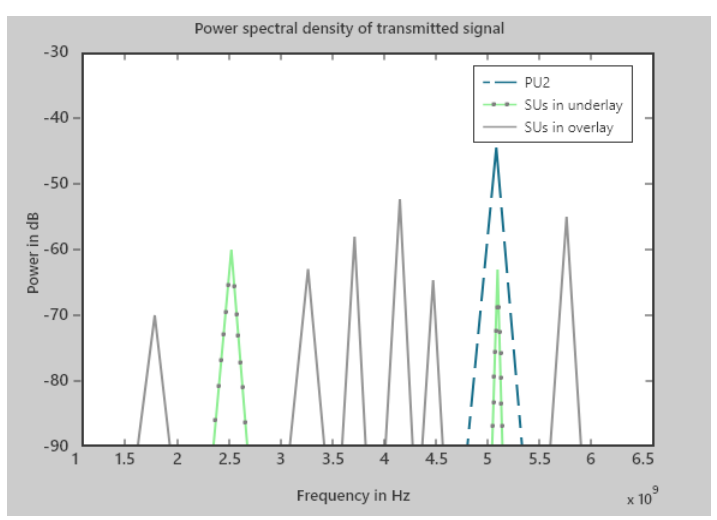

(b)

Figure 10. SU-Tx1 and SU-Tx2 are accessing the channel in absence of PU1 at their respective time slots (a) SU-Tx1 accessing channel with lower BW and (b) SU-Tx2 accessing channel with higher BW one at a time

SU-Tx 1 has slower BW adaptation rate of 0.5 with low subcarrier density with subcarrier spacing of 9.765625 KHz occupies BW of $22.5 \mathrm{MHz}$ and SU-Tx2 has faster BW adaptation rate of 0.75 with high subcarrier density with subcarrier $4.8828 \mathrm{kHz}$ occupies BW of $32.5 \mathrm{MHz}$ at Iteration 1. At iteration 4 SU-Tx2 due to higher data requirements and faster learning rate attains the synchronization where SU Tx1 still has not acquired the full bandwidth of PU1. The BW adaptation of SU-Tx1 continues till iteration 7 when it also attains synchronization. The basic idea of this bandwidth adaptation is that each SU would be allowed a complete access to the PU band in its absence at individual time instants. The Table 8 details the different bandwidth reconfiguration steps of each of SUs: SU-Tx1 and SU-Tx2 at different time instants, along with their individual data rates achieved and IT caused. As shown in Table 9 IT difference accounts for SUs to increase their signal strengths in case of different positions of their receiving units in order to further enhance the data rate and achieve the maximum capacity of the channel. It leads to power reconfiguration of SUs Tx1 and Tx2 at respective time slots.

Now SU-Tx1 has higher data rate demands therefore its transmitter reconfigures the power with a faster learning rate of 0.75 while SU2 has lower rate of power reconfiguration of 0.5 . Both SUs continues increasing their powers in alternate time slots at each iteration. Power locking condition is met for the SU1 early whose data rate demands are satisfied at a faster rate while SU2 is in reconfiguration state since its receiver demands of higher rate at relatively lower distance than that of SU1 for prolonged duration. It can be concluded that both the SUs: SU-Tx1 and SU-Tx2 attain the limits of IT individually and satisfy their receiver demands rendering 238.135 Mbps and 459.96 Mbps data rates respectively as shown in Figure 11 . The Table 10 underneath details the different power reconfiguration steps of each of SUs: SU-R1 and SU-R2 at different time instants, along with their individual data rates achieved and IT caused.

The Table 11 accounts for the data rates achieved individually by SU-Tx1 and SU-Tx2 at their respective time instants. The IT limit can be individually attained by each of the user and the entire spectrum of PU can be fully allocated to each user at particular instant. Figure 12 shows the data rate achieved during power reconfiguration of SU-Tx1 and SU-Tx2. It is clear that due to SU-Tx1 attains higher data rate in less time as compared to SU-Tx2 due to significant rise in its signal strength. 
Table 8. Bandwidth adaptation steps and IT and data rate calculation

\begin{tabular}{cccccc}
\hline Time slot & $\begin{array}{c}\text { Reconfigured } \\
\text { SU }\end{array}$ & $\begin{array}{c}\text { Reconfigured bandwidth } \\
\text { MHz }\end{array}$ & POWER in dB & Data rate mbps & $\begin{array}{c}\text { IT } \\
\text { dB K }\end{array}$ \\
\hline T1 & SU-Tx1 & 22.5 & 30 & 92.1 & 310 \\
T2 & SU-Tx2 & 32.5 & 40 & 309.3 & 346 \\
T3 & SU-Tx1 & 31.25 & 30 & 128 & 352 \\
T4 & SU-Tx2 & 38.1250 & 40 & 362.6 & 424 \\
T5 & SU-Tx1 & 35.6250 & 30 & 145.92 & 419 \\
T6 & SU-Tx2 & 39.5313 & 40 & 375.4 & 488 \\
T7 & SU-Tx1 & 37.8125 & 30 & 154.9 & 453 \\
T8 & SU-Tx2 & $39.8828($ SYNC) & 40 & 379.8 & 526 \\
T1 & SU-Tx1 & 38.9063 & 30 & 159.4 & 470 \\
T2 & SU-Tx1 & 39.7256 & 30 & 162.7 & 470 \\
T3 & SU-Tx1 & 40(SYNC) & 30 & 163.4 & 487 \\
\hline
\end{tabular}

Table 9. Parameters of SU1 and SU2 after BW synchronization

\begin{tabular}{ccccccc}
\hline SU & $\begin{array}{c}\text { BW } \\
\text { MHz }\end{array}$ & $\begin{array}{c}\text { POWER } \\
\text { dB }\end{array}$ & $\begin{array}{c}\text { Data rate } \\
\text { mbps }\end{array}$ & $\begin{array}{c}\text { Achievable capacity } \\
\text { Mbps }\end{array}$ & $\begin{array}{c}\text { IT } \\
\text { db K }\end{array}$ & IT diff. \\
\hline SU-Tx1 & 40 & 30 & 163.8 & 240 & 2899 & 602 \\
SU-Tx2 & 40 & 40 & 379.8 & 460 & 2938 & 562 \\
\hline
\end{tabular}

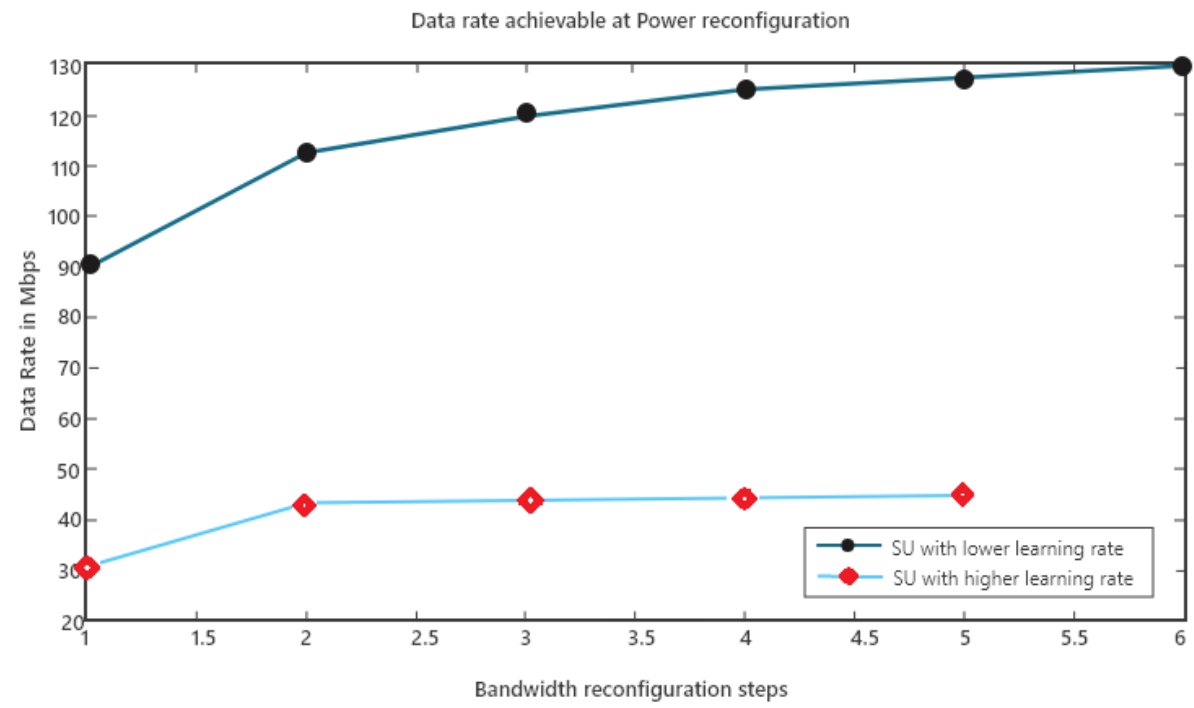

Figure 11. Data rates achieved after BW adaptation

Table 10. Power reconfiguration step: IT and data rate calculations

\begin{tabular}{cccccc}
\hline Time slot & $\begin{array}{c}\text { Reconfigured } \\
\text { SU }\end{array}$ & $\begin{array}{c}\text { BW } \\
\text { MHz }\end{array}$ & $\begin{array}{c}\text { Reconfigured POWER in dB } \\
\text { Data rate mbps }\end{array}$ & $\begin{array}{c}\text { IT in } \\
\text { dB K }\end{array}$ \\
\hline T1 & SU-Tx1 & 40 & 56.25 & 203.8 & 677 \\
T2 & SU-Tx2 & 40 & 50 & 423.5 & 685 \\
T3 & SU-Tx1 & 40 & 62.8125 & 234.6 & 822 \\
T4 & SU-Tx2 & 40 & 55 & 442.4 & 825 \\
T5 & SU-Tx1 & 40 & 64.45 & 237.2 & 935.9 \\
T6 & SU-Tx2 & 40 & 57.5 & 451.3 & 936.1 \\
T7 & SU-Tx1 & 40 & $65($ SYNC) & 238.1 & 1081 \\
T8 & SU-Tx2 & 40 & 58.12 & 455.7 & 1026 \\
T1 & SU-Tx2 & 40 & 58.75 & 457.8 & 1065 \\
T2 & SU-Tx2 & 40 & $59.92($ SYNC) & 459.9 & 1083 \\
\hline
\end{tabular}

Table 11. Data rates and IT achieved by SU1 and SU2 at independent time instants after reconfiguration

\begin{tabular}{ccccccc}
\hline SU & $\begin{array}{c}\text { BW } \\
\text { MHz }\end{array}$ & $\begin{array}{c}\text { POWER } \\
\text { in dB }\end{array}$ & $\begin{array}{c}\text { Data rate } \\
\text { MBPS }\end{array}$ & $\begin{array}{c}\text { Achievable } \\
\text { capacity Mbps }\end{array}$ & $\begin{array}{c}\text { TOTAL IT in } \\
\text { dB Kelvin }\end{array}$ & $\begin{array}{c}\text { IT } \\
\text { DIFF. }\end{array}$ \\
\hline SU-Tx1 & 40 & 65 & 238.1 & 240 & 3493 & 7 \\
SU-Tx2 & 40 & 59.92 & 459.9 & 460 & 3495 & 5 \\
\hline
\end{tabular}




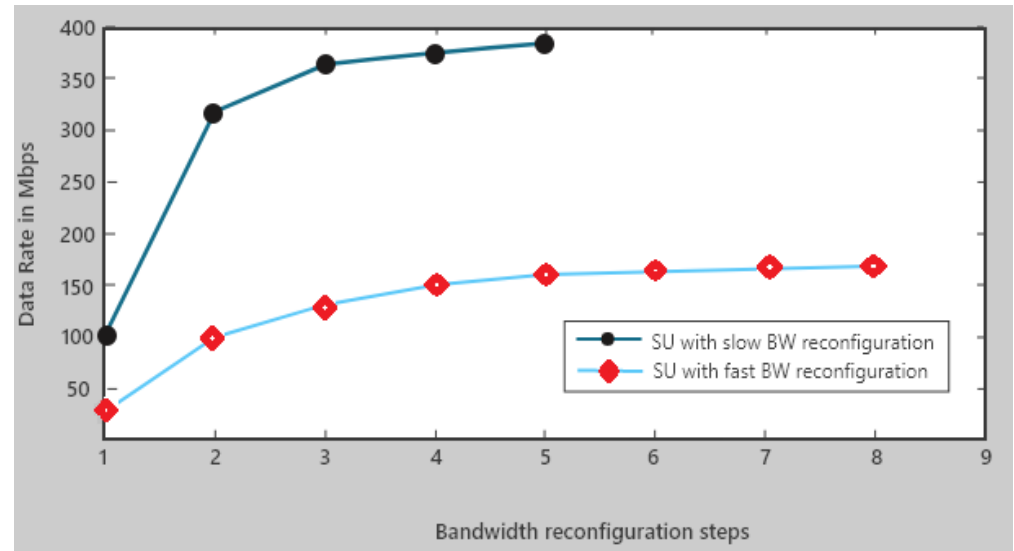

Figure 12. Data rates achieved by power reconfiguration of SU1 and SU2 at alternate time instants

\section{RESULT}

\subsection{Transmit power control}

The proposed method reconfigures the transmit power of secondary users according to the data rate requirements of the secondary receivers. When $\mathrm{S} / \mathrm{N}$ ratio at the receivers is sufficiently above the threshold required for efficient communication, it is beneficial to assign the power levels according to the need of the secondary users. After bandwidth adaptation when secondary transmitters have achieved the bandwidth that of PU1 and there is no more scope of data rate enhancement then according to Shannon theorem further data rate can be enhanced by increasing the transmit power levels. The proposed system assigns the power level according to the data rate requirement of secondary receivers. The users having higher data requirements are assigned higher power levels iteratively and vice-versa. Figure 13 shows the power reconfiguration at different iterations for FDMA system. After bandwidth adaptation the transmit power of both SU-Tx1 and SU-Tx2 was $30 \mathrm{dBm}$. But as the power reconfiguration begins due to higher data rate requirement of SU-Rx2 was assigned higher power levels were assigned to SU-Tx2 as compared SU-Tx1. Figure 14 shows the power reconfiguration for TDMA case. During bandwidth adaptation SU-Tx1 and SU-Tx2 were transmitting at $30 \mathrm{dBm}$ and $40 \mathrm{dBm}$ respectively and data rate requirement of SU-Rx2 were higher than SU-Rx1. As the power reconfiguration begins data rate requirement of SU- Rx1 becomes higher than SU-Rx2, so SU-Tx1 were assigned higher power levels as compared to SU-Tx2.

Figure 15 shows the comparison of peak power per node of Primary user PU1 and the SU-Tx1 and SU-Tx2 transmitting in the absence of PU1 for TDMA case and FDMA case. The graph shows that SU1 and SU2 are adjusting their transmit powers according to the data requirements of their corresponding receivers depending upon their feedback whereas due to no feedback PU1 keeps transmitting at fixed power. The proposed feedback system can optimize the transmit power of secondary transmitters according to the data requirements of their receivers respectively.

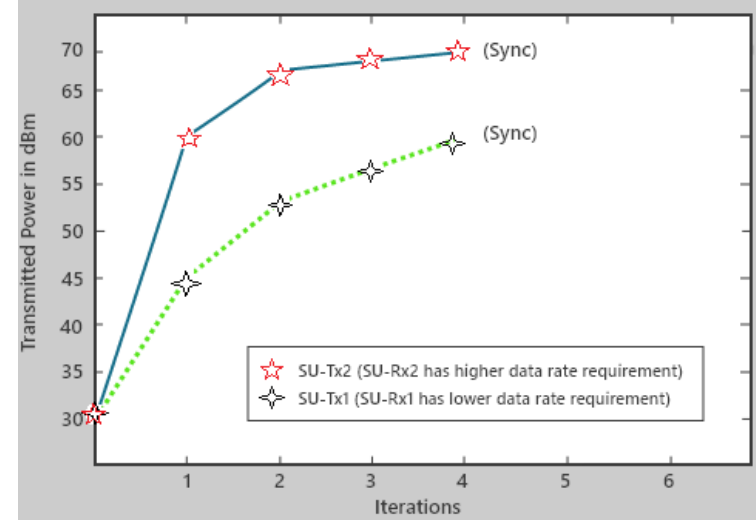

Figure 13. Variation of transmit power at each each iteration for FDMA

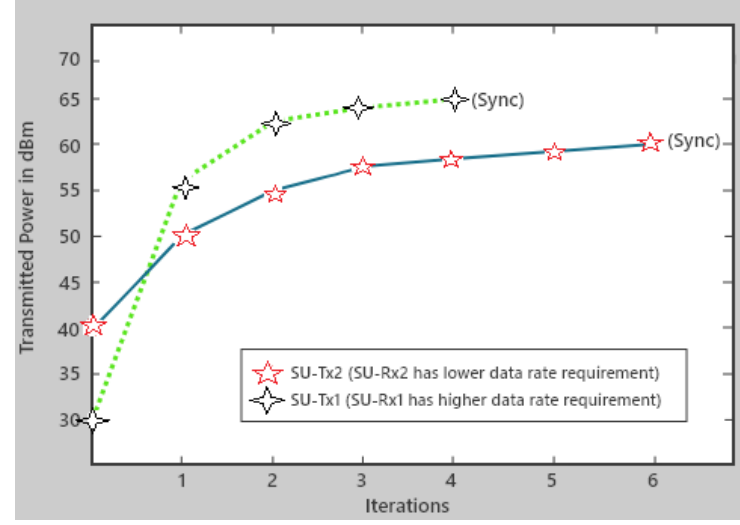

Figure 14. Variation of transmit power at each iteration for TDMA 


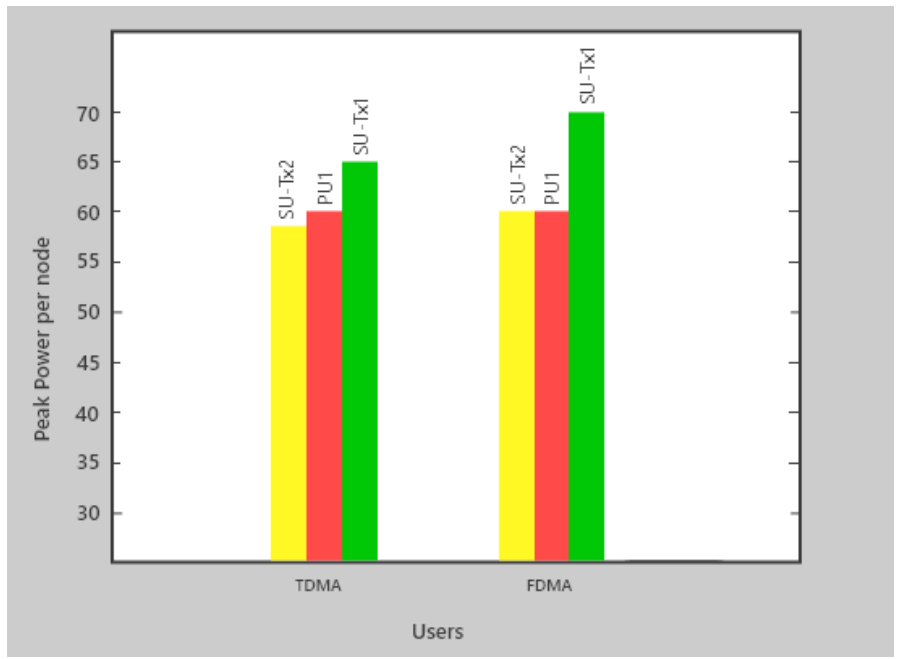

Figure 15. Compares the peak power per node of SU-Tx1, SU-Tx2 and PU1 for TDMA and FDMA case

\subsection{Analysis in fading environments}

The results of the proposed technique are analyzed in fading scenario other than AWGN. The Rayleigh and Nakagami fading [22]-[26] $(\mathrm{M}=2)$ channels are selected. The fading channels are implemented using communication tool box in MATLAB. Both the channels are considered to analyze the performance of proposed technique in multipath scattering effects, time dispersion, and Doppler shifts that arise from relative motion between the transmitter and receiver. Sampling rate of $1000 \mathrm{~Hz}$ and maximum Doppler shift of $30 \mathrm{~Hz}$ is selected for Rayleigh fading channel. Frequency flat Rayleigh fading is considered. The probability density function of channel power gain is given as in (6).

$$
g(x)=\frac{x}{\sigma^{2}} e^{-\frac{x^{2}}{2 \sigma^{2}}} \quad, \mathrm{x}>0
$$

For a unit-mean Nakagami $\mathrm{M}$ fading channel the PDF of channel power gains follow gamma distribution as in (7). The fading parameter $\mathrm{M}$ is the ratio of the power of line-of-sight signal to that of the multi-path component. When $\mathrm{M}=1$ the channel behaves like Rayleigh fading channel and for $\mathrm{M}=\infty$ the channel behaves like AWGN. We have considered $M=2$ Nakagami fading.

$$
g(x)=\frac{M^{M} x^{(M-1)}}{\gamma(M)} e^{-M x} \quad, \mathrm{x}>0
$$

The analysis of proposed technique in various fading environments shows that in Rayleigh fading which is worst fading scenario, due to deep fades between Primary users and secondary users, the effect of interference of SU to PU is less as a result SU-Tx's gets the opportunity to raise their transmit power levels before maximum interference temperature limit is reached. As a result, the capacity of the SU-Tx1 and Su-Tx2 is further enhanced till a certain point. After that data rate becomes almost constant. Figure 16(a) shows the transmit power versus data rate curves of SU-Tx1 and SU-Tx2 for FDMA case. As both secondary users transmit simultaneously there is small rise in data rate due to increase transmit power. Figure 16(b) shows the transmit power versus data rate curves of SU-Tx1 and SU-Tx2 for TDMA case.

\subsection{Comparison of the feedback model with system without feedback}

Figure 17(a) shows the comparison of the proposed technique of multiple secondary users accessing the unused bandwidth of Primary users with that of system without feedback. This system is $43 \%$ to $95 \%$ more efficient than the existing system. The proposed system work on demand and supply concept with feedback from the secondary receivers is of utmost importance.

\subsection{Comparison of bandwidth access using FDMA and TDMA}

Figure 17(b) gives the comparison of two cases where in first case the two secondary users access the bandwidth of PU1 in its absence simultaneously (FDMA) and in other case two SUs accesses the bandwidth one by one at their designated time slot (TDMA). It is found that in TDMA (case2) achieves higher data rates over a particular bandwidth i.e., TDMA has higher spectral efficiency (R/BW) as compared to FDMA (case 1) 
because FDMA divides the rate between the multiple users it withstands simultaneously while in TDMA a whole access to the band is allowed for an individual user at particular time instant.

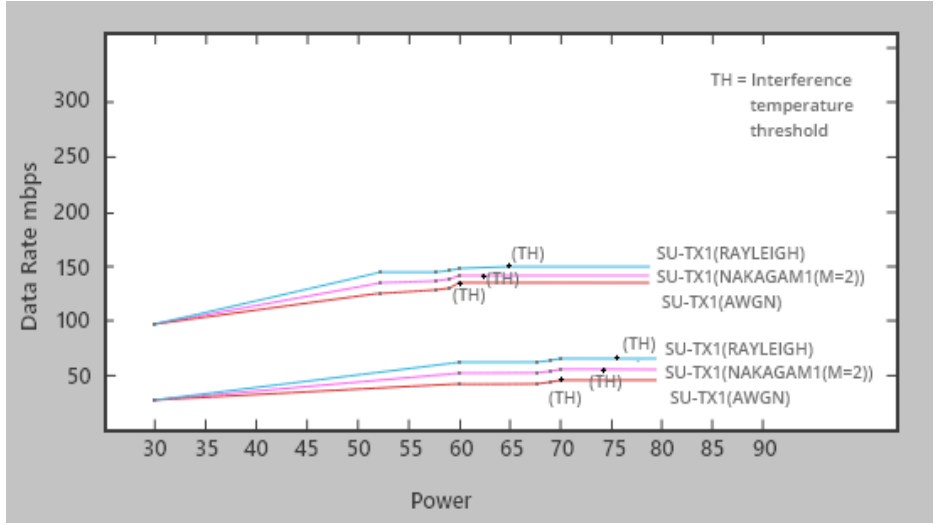

(a)

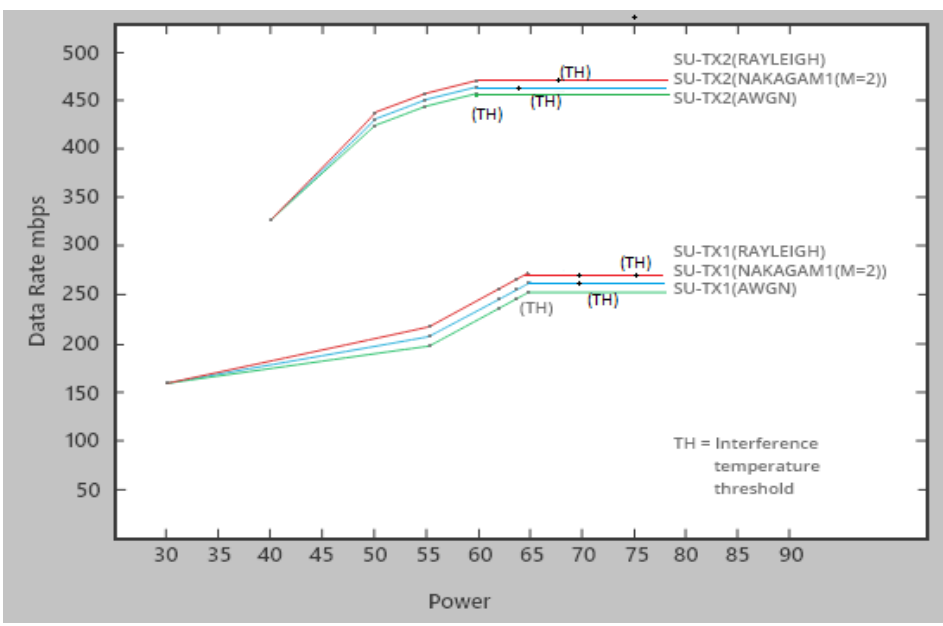

(b)

Figure 16. Power v/s data rate for SU-Tx1 and SU-Tx2 for AWGN, Nakagami (M=2) and Rayleigh fading channel for (a) FDMA and (b) TDMA

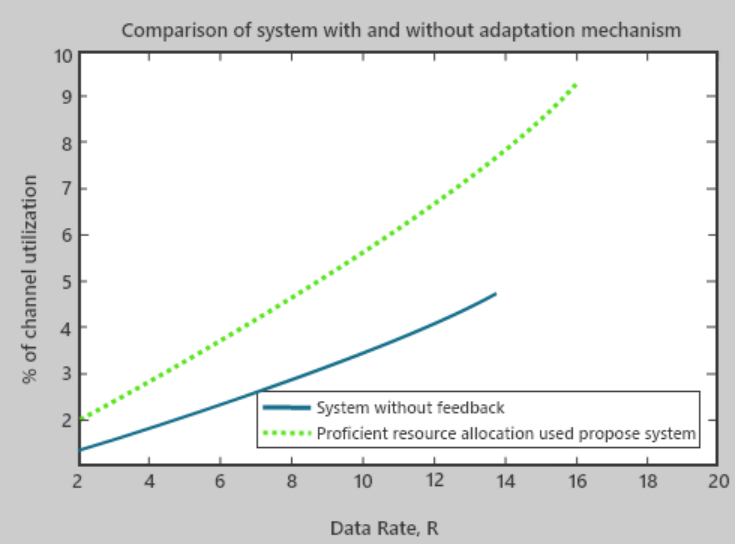

(a)

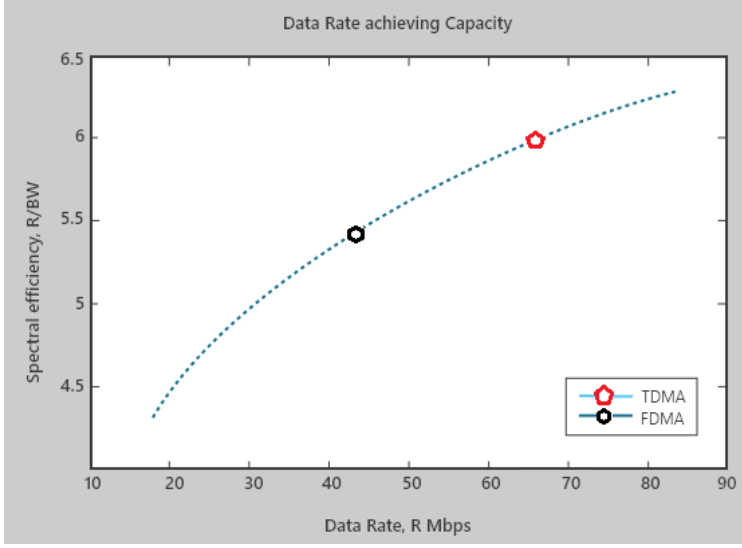

(b)

Figure 17. Comparison of (a) proposed system with system without feedback and (b) TDMA and FDMA system of spectrum access by two SU's 


\subsection{Comparison of proposed method with the existing techniques}

The proposed technique using TDMA and FDMA access is compared with existing techniques on the basis of channel utilization efficiency per user w.r.t transmit power as shown in Figure18. The proposed method optimizes the resources like power, bandwidth, and data rate efficiently by observing the performance of the time frames when the data has been transferred through observed PU data transfer and SU data transfer and creating repository of 100,000 simulations analyzing the data to be on true side or on the false side. The optimized transmit power aids in increasing the channel efficiency by adding extra bits before interference temperature limit is reached. The existing techniques are Chu's algorithm, Lee's algorithm and Majidi's algorithm as discussed in [24]-[26]. The FDMA access method used in proposed technique shows minimum channel utilization per user as compared to all techniques. It is because FDMA divides the rate between the multiple users it withstands simultaneously. The TDMA access technique in proposed method give maximum channel utilization efficiency of $99.5 \%$ as compared to other techniques but it uses higher power levels to achieve the same. Where Chu's algorithm achieves maximum channel utilization efficiency at $50 \mathrm{~dB}$, the TDMA access technique achieves it at $60 \mathrm{~dB}$. The proposed method improves the spectral efficiency by $4.5 \%$ as compared to existing techniques and achieved high data rate up to $460 \mathrm{Mbps}$.

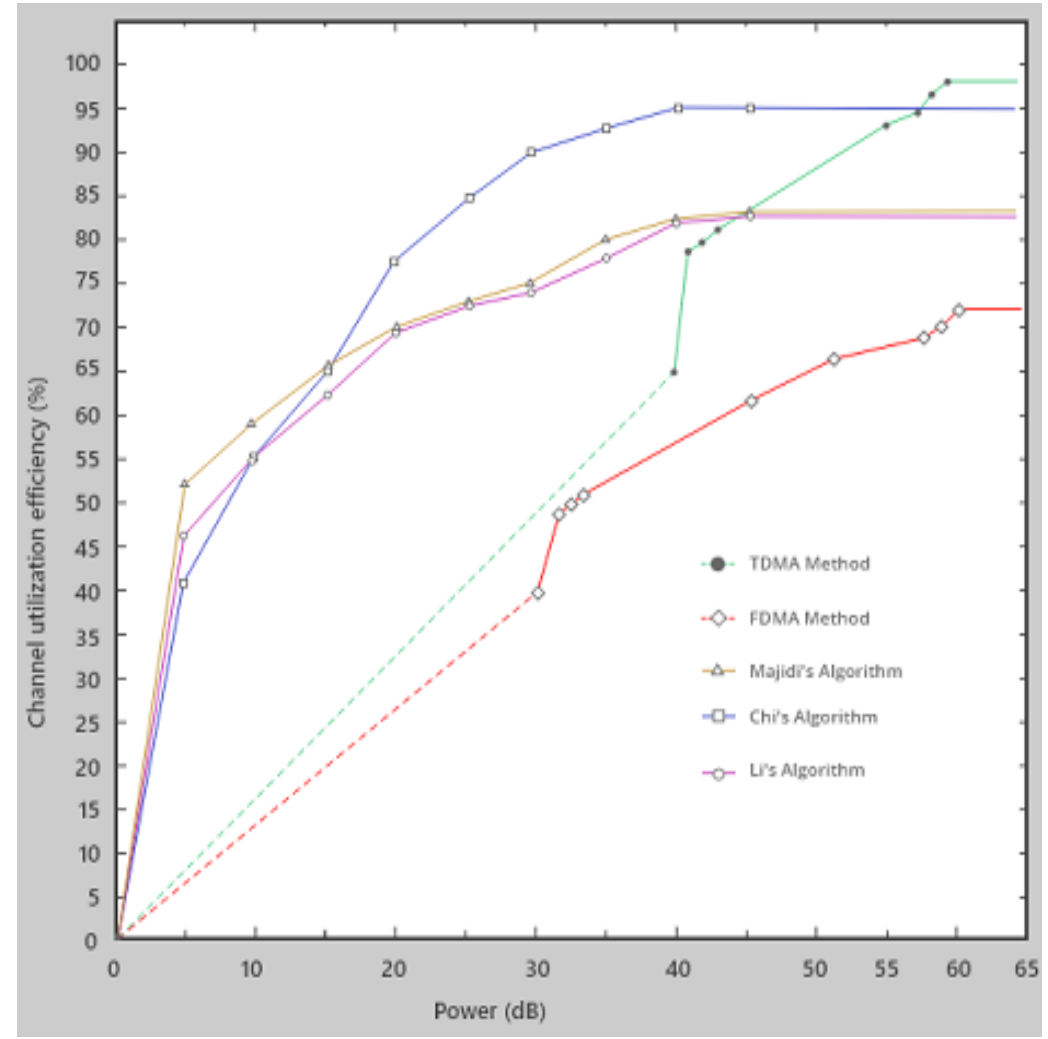

Figure 18. Comparison of proposed method with the existing techniques

\section{CONCLUSION}

The proposed system once again shows that cognitive radio is a useful technology to overcome the problem of underutilization of licensed spectrum. The proposed feedback system proves its utility by efficiently utilizing the spectrum as compared to existing system without feedback. More no. of users was able to access the network at a time in the absence of licensed users. Reconfiguration of secondary users in bandwidth and transmit power helped achieve higher data rates and also achieve improved range for long distance communication respectively. The secondary transmitters were able to adjust their transmit powers according to the data rate requirements of secondary receivers and by the employment of computational intelligence technique, use of transmit power was optimized. Optimization of transmit power helped in achieving the $4.5 \%$ higher spectral efficiency as compared to the existing techniques. In future work re-arrival of licensed users can be discussed and readjustment of parameters can be discussed when PU reclaim its band. Other spectrum sensing methods can be used to overcome noise uncertainties in the channel. 


\section{ACKNOWLEDGMENT}

The author is highly thankful to IK Gujral Punjab Technical University, Kapurthala for giving the opportunity to carry out the research work and providing the resources as and when required.

\section{REFERENCES}

[1] P. Kolodzy and I. Avoidance, "Spectrum policy task force," International Symposium on Advanced Radio Technologies. Federal Commun. Comm., Washington, DC, Rep. ET Docket, pp. 1-14, 2002.

[2] J. Mitola and G. Q. Maguire, "Cognitive radio: making software radios more personal," IEEE Personal Communications, vol. 6, no. 4, pp. 13-18, 1999, doi: $10.1109 / 98.788210$.

[3] T. Clancy and W. Arbaugh, "Measuring interference temperature," Virginia tech symposium on wireless personal communications, no. $1,2006$.

[4] P. Setoodeh and S. Haykin, "Robust transmit power control for cognitive radio," Proceedings of the IEEE, vol. 97, no. 5, pp. 915-939, May 2009, doi: 10.1109/JPROC.2009.2015718.

[5] A. Sharma, V. Hastir, and D. S. Saini, "Transmit power optimization in cognitive radio networks using game theoretic approach," in 2014 International Conference on Signal Propagation and Computer Technology (ICSPCT 2014), 2014, pp. 312-316, doi: 10.1109/ICSPCT.2014.6884889.

[6] K. Umebayashi, J. Lehtomäki, and I. M. Suliman, "Analysis of transmit power setting technique for cognitive radio networks," Eurasip Journal on Wireless Communications and Networking, vol. 2016, no. 1, Jun. 2016, doi: 10.1186/s13638-016-0638-9.

[7] P. Goel, A. Singh, and A. Goel, "A survey on optimization of transmit power in cognitive radio networks through nature inspired computational intelligence techniques," ICTACT Journal on Communication Technology, vol. 21, no. 1, pp. 259-2264, 2021, doi: 10.21917/ijct.2021.0348

[8] N. Benghabrit and M. Kaddour, "Optimizing the capacity of cognitive radio networks with power control and variable spectrum allocation," Transport and Telecommunication Journal, vol. 19, no. 2, pp. 128-139, Jun. 2018, doi: 10.2478/ttj-2018-0011.

[9] C. S. Preetham, M. S. G. Prasad, D. S. S. L. Saranya, C. T. Somepalli, D. B. S. Sai Krishna, and V. Rohit, "Performance analysis of cooperative hybrid cognitive radio network with various diversity techniques," International Journal of Electrical and Computer Engineering (IJECE), vol. 6, no. 5, p. 2125, Aug. 2016, doi: 10.11591/ijece.v6i5.pp2125-2133.

[10] M. Tuberquia and C. Hernandez, "New approaches in cognitive radios using evolutionary algorithms," International Journal of Electrical and Computer Engineering (IJECE), vol. 8, no. 3, p. 1636, Jun. 2018, doi: 10.11591/ijece.v8i3.pp1636-1646.

[11] A. M, S. Vemuru, and T. Gunasekhar, "Transmission protocols in cognitive radio mesh networks," International Journal of Electrical and Computer Engineering (IJECE), vol. 5, no. 6, p. 1446, Dec. 2015, doi: 10.11591/ijece.v5i6.pp1446-1451.

[12] Madhavi B Dubal and S. A. Shirsat., "OFDM modulator for wireless LAN (WLAN)," International Journal of Scientific \& Engineering Research, vol. 4, no. 8, 2013, Art. no. 1166

[13] H. Malik, D. R. Rotake, and M. Mahajan, "Design and Implementation of Bpsk modulator and demodulator using VHDL," IOSR Journal of Electronics and Communication Engineering, vol. 9, no. 3, pp. 98-105, 2014, doi: 10.9790/2834-093498105.

[14] S. P. Yadav and S. C. Bera, "Single carrier FDMA technique for wireless communication system," in 2015 Annual IEEE India Conference (INDICON), 2015, pp. 1-6, doi: 10.1109/INDICON.2015.7443655.

[15] D. D. Falconer, F. Adachi, and B. Gudmundson, "Time division multiple access methods for wireless personal communications," IEEE Communications Magazine, vol. 33, no. 1, pp. 50-57, 1995, doi: 10.1109/35.339881.

[16] E. Axell, G. Leus, and E. G. Larsson, "Overview of spectrum sensing for cognitive radio," in 2010 2nd International Workshop on Cognitive Information Processing, 2010, pp. 322-327, doi: 10.1109/CIP.2010.5604136.

[17] S. Reel and A. K. Goel, "Artificial neural networks and fuzzy logic in process modeling and control," Communications in Computer and Information Science, vol. 250 CCIS, pp. 808-810, 2011, doi: 10.1007/978-3-642-25734-6_144.

[18] M. Sharma, A. Sahoo, and K. D. Nayak, "Channel modeling based on interference temperature in underlay cognitive wireless networks," in 2008 IEEE International Symposium on Wireless Communication Systems, 2008, pp. 224-228, doi: 10.1109/ISWCS.2008.4726051.

[19] M. Franceschetti, M. D. Migliore, and P. Minero, "The capacity of wireless networks: information-theoretic and physical limits," IEEE Transactions on Information Theory, vol. 55, no. 8, pp. 3413-3424, Aug. 2009, doi: 10.1109/TIT.2009.2023705.

[20] A. K. Goel, S. C. Saxena, and S. Bhanot, "A fast learning algorithm for training feedforward neural networks," International Journal of Systems Science, vol. 37, no. 10, pp. 709-722, Aug. 2006, doi: 10.1080/00207720600799468.

[21] K. Zheng, X.-Y. Liu, X. Liu, and Y. Zhu, "Hybrid overlay-underlay cognitive radio networks with energy harvesting," IEEE Transactions on Communications, vol. 67, no. 7, pp. 4669-4682, Jul. 2019, doi: 10.1109/TCOMM.2019.2912605.

[22] S. Popa, N. Draghiciu, and R. Reiz, "Fading types in wireless communications systems," Journal of Electrical and Electronics Engineering, vol. 1, no. 1, pp. 232-237, 2008.

[23] A. A. Rosas, A. M. Benaya, M. Shokair, and S. A. El-dolil, "Optimal power allocation technique using genetic algorithm for maximization of Ergodic capacity under different fading channel approaches in cognitive radio network," in 201633 rd National Radio Science Conference (NRSC), 2016, vol. 2016-April, pp. 132-140, doi: 10.1109/NRSC.2016.7450846.

[24] H. Chu, L. Xu, and Y. Liu, "An optimal power allocation algorithm for cognitive radio networks based on maximum rate and interference constraint," Ingénierie des systèmes d information, vol. 24, no. 2, pp. 155-159, Jul. 2019, doi: 10.18280/isi.240204.

[25] M. Majidi, A. Mohammadi, and A. Abdipour, "Analysis of the power amplifier nonlinearity on the power allocation in cognitive radio networks," IEEE Transactions on Communications, vol. 62, no. 2, pp. 467-477, Feb. 2014, doi: 10.1109/TCOMM.2014.011114.130094.

[26] L. Li, X. Zhou, H. Xu, G. Y. Li, D. Wang, and A. Soong, "Simplified relay selection and power allocation in cooperative cognitive radio systems," IEEE Transactions on Wireless Communications, vol. 10, no. 1, pp. 33-36, Jan. 2011, doi: 10.1109/TWC.2010.101810.100311. 